\title{
Indicadores de obesidade central como preditores de excesso de peso em crianças e adolescentes
}

\author{
Central obesity indicators as predictors of weight in \\ children and adolescents
}

\author{
Mateus Augusto Bim ${ }^{1}$ \\ Deonilde Balduíno ${ }^{2}$ \\ Sandro Claro Pedrozo ${ }^{2}$
}

\section{RESUMO}

Introdução: As prevalências de sobrepeso e obesidade mundial aumentaram significativamente em crianças e adolescentes. Indicadores antropométricos são amplamente utilizados para triagem de indivíduos com maiores riscos à saúde. Objetivo: 0 objetivo deste estudo foi verificar a capacidade de indicadores antropométricos de obesidade central como preditores de excesso de peso em crianças e adolescentes. Trata-se de um estudo transversal, que investigou 924 escolares de seis a 13 anos. Foram coletadas as medidas de massa corporal, estatura e perímetro da cintura para determinar o excesso de peso (índice de massa corporal), o perímetro da cintura, a razão cintura estatura e o índice de conicidade. Para análise do desempenho diagnóstico dos indicadores de obesidade central em predizer excesso de peso foram determinadas as áreas sobre a curva ROC. Resultados: Todos os indicadores demonstraram boa capacidade em predizer excesso de peso em crianças e adolescentes, com exceção do índice de conicidade em crianças do sexo masculino. Conclusão: 0 perímetro da cintura e a razão cintura estatura demonstraram as maiores áreas sobre a curva ROC. Os indicadores de obesidade central abdominal devem ser incluídos na avaliação antropométrica de professores de educação física para identificar crianças e adolescentes com maiores riscos de saúde.

\section{PALAVRAS-CHAVE}

Obesidade pediátrica; Antropometria; Saúde da criança; Saúde do adolescente.

\footnotetext{
${ }^{1}$ Universidade do Estado de Santa Catarina (UDESC).

${ }^{2}$ Universidade do Oeste de Santa Catarina (Unoesc).
} 


\section{ABSTRACT}

Introduction: The prevalence of overweightando besity worldwide increased significantly in children and adolescents. Anthropometric indicators are widelyusedtoscreenindividual swithhigher healthrisks. Objective: The aim of this study was to verify the ability of anthropometric indicators of central obesity as predictors of overweight in children and adolescents. This is a cross-sectional study, which investigated 924 schoolchildren aged six to 13 years. Body mass, height and waist circumference measurements were collected to determine excess weight (body mass index), waist circumference, waist-to-height ratio and conicity index. To analyze the diagnostic performance of central obesity indicators in predicting excess weight, the areas on the ROC curve were determined. Results: All indicators showed a good ability to predict overweight in children and adolescents, with the exception of the taper index in male children. Waist circumference and waist-to-height ratio showed the largest areas on the ROC curve. Conclusion: Central obesity indicators should be included in the anthropometric assessment of physical education teachers to identify children and adolescents with the greatest health risks.

\section{KEYWORDS}

Pediatric obesity; Anthropometry; Child health; Adolescent health. 


\section{INTRODUÇÃo}

O excesso de peso se tornou um sério problema de saúde pública em grande parte do mundo (ABARCA-GÓMEZ et al., 2017). Nas últimas décadas têm sido observados aumentos expressivos da obesidade central em populações pediátricas. Estima-se que entre 1975 e 2016 o número de meninas obesas central aumentou de $5 \mathrm{mi}$ lhões para 50 milhões e nos meninos de 6 milhões para 74 milhões (ABARCA-GÓMEZ et al., 2017). Evidências apontam que ser obeso na infância aumenta as chances de se manter obeso na vida adulta, especialmente a obesidade na adolescência (SIMMONDS et al., 2016).

Devido ao crescimento da obesidade a nível mundial, pesquisadores têm utilizado indicadores antropométricos para avaliar a composição corporal de crianças e adolescentes em estudos epidemiológicos (PELEGRINI et al., 2015; WEISS et al., 2014; DUMITH et al. 2018). Apesar de não apresentarem a mesma precisão dos métodos avançados, como a absorciometria por dupla emissão de raios-X (DXA) e a pletismografia por deslocamento de ar (PDA), os indicadores antropométricos têm sido amplamente utilizados por pesquisadores em estudos de grande escala pelo baixo custo, fácil aplicabilidade e interpretação (ALVES JÚNIOR et al., 2017) e boa sensibilidade e especificidade para determinar gordura corporal elevada (PELEGRINI et al., 2015).

Diversos indicadores antropométricos têm sido empregados para discriminar adolescentes com gordura corporal elevada (PELEGRINI et al., 2015; WEISS et al., 2015), obesidade abdominal (CARVALHO et al., 2018), excesso de peso (DUMITH et al., 2018.) e pressão arterial elevada (KUCIENE; DULSKIENE, 2019). No entanto nenhum estudo comparou qual o melhor indicador antropométrico de obesidade abdominal para predizer excesso de peso em crianças e adolescentes. Dessa forma, são necessários mais estudos que investiguem o poder diagnóstico desses indicadores, especialmente em populações pediátricas, visando a importância da prevenção e tratamento da obesidade em idades tenras.

Neste sentido, o objetivo deste estudo foi verificar a capacidade de indicadores antropométricos de obesidade central como preditores de excesso de peso em crianças e adolescentes.

\section{MATERIAIS E MÉTODO}

Este estudo, de delineamento transversal incluiu crianças e adolescentes matriculados no ensino fundamental nas escolas públicas da rede municipal de Xanxerê, Santa Catarina. Fizeram parte do estudo escolares de 9 escolas, sendo que todos os alunos de seis a 13 anos foram convidados para participar do estudo. Para aplicação da pesquisa foram seguidos os critérios éticos de acordo com os padrões de normas e diretrizes regulamentadoras da pesquisa envolvendo seres humanos, em conformidade com a Resolução 466, de 2012, do Conselho Nacional de Saúde (MINISTÉRIO DA SAÚDE; CONSELHO NACIONAL DE SAÚDE, 2013) e com a Declaração de Helsinki.

Foram considerados elegíveis para o estudo as crianças e adolescentes que: 1) entregaram o termo de assentimento (TA) assinado pelos mesmos e o termo de consentimento livre e esclarecido (TCLE) assinado pelos pais ou responsável; 2) estavam regularmente matriculados nas escolas públicas da rede municipal de Xanxerê, SC; 3) que estavam presente no dia em que foram realizadas as coletas; 4) com seis a 13 anos de idade; e 5) que possuíam os dados de massa corporal, estatura e perímetro da cintura coletados.

Para determinar a relação cintura/estatura (RCE), o índice de massa corporal (IMC) e o perímetro da cintura (PerC) foram utilizadas as medidas de massa corporal, estatura e perímetro de cintura.

A massa corporal foi mensurada por uma balança digital da marca Ultra Slim Wiso (100g de resolução), com o avaliado em pé sob a balança e olhar voltado para frente. A estatura foi mensurada por um estadiômetro portátil da marca Alturexata $(0,1 \mathrm{~cm}$ de resolução), com o avaliado em pé, descalço, de costas para o estadiômetro, com a cabeça posicionada no plano de Frankfort e os calcanhares, a região glútea e a região occipital do crânio tocando o estadiômetro.

0 índice de massa corporal (IMC) foi determinado pela equação: peso $(\mathrm{kg})$ / estatura ${ }^{2}(\mathrm{~m})$. Para categorização foram utilizados os critérios recomendados pela International Obesity Task Force (IOTF) (COLE et al., 2000) específicos para sexo e idade, que classificam crianças e adolescentes com sobrepeso e obesidade. Para este estudo foram considerados com excesso de peso os sujeitos com sobrepeso e obesidade. 
O perímetro da cintura (PerC) foi mensurado por uma fita antropométrica inelástica da marca Sanny (resolução de 0,1 mm). A medida foi realizada horizontalmente no ponto médio entre a borda inferior do último arco costal e a crista ilíaca, com o avaliado em posição ortostática e abdômen relaxado (FERNANDES FILHO 2003).

A razão cintura estatura (RCE) foi calculada pela divisão entre o perímetro da cintura pela estatura. 0 índice de conicidade (Indice C) foi calculado pela equação perímetro da cintura $(\mathrm{m}) / 0,109 *(\sqrt{\text { peso corporal }}(\mathrm{kg}) /$ estatura (m)).

As análises foram realizadas em dois grupos, o grupo constituído por crianças ( 6 a 9 anos de idade) e o grupo constituído por adolescentes (10 a 13 anos de idade). Os grupos foram formados de acordo com a Organização Mundial da Saúde (WHO, 2005) que considera indivíduos de 10 a 19 anos como adolescentes.

Para as análises descritivas foram utilizadas as médias, desvio padrão e frequências. A normalidade dos dados foi verificada pelo teste Kolmogorov-Smirnov. Para comparação de médias foi realizado o teste $U$ de Mann-Whitney e o teste t para amostras independentes.

O desempenho diagnóstico dos indicadores de obesidade central na detecção de excesso de peso foi avaliado pela análise da curva ROC (Receiver Operating Characteristic). Esta análise refere-se à capacidade do PerC, da RCE e do Índice C em discriminar indivíduos com excesso de peso. Para isso, foram determinadas as áreas sobre a curva ROC e seus respectivos intervalos de confiança de 95\% (IC95\%), bem como a sensibilidade e especificidade de cada indicador de obesidade central e os IC95\%. Para demonstrar poder discriminatório a área sobre a curva ROC precisa apresentar valor acima de 0,50 (limite não discriminatório). Quanto mais próxima de 1,0 (discriminação perfeita), melhor a área sobre a curva ROC e melhor será a capacidade de discriminação. Sendo assim, melhores valores são considerados a partir de uma área sobre a curva ROC de 0,75 (CERDA; CIFUENTES, 2012).

Os procedimentos estatísticos foram realizados no software IBM SPSS Statistics 20.0 e no MedCalc com nível de significância estabelecido em $\mathrm{p}<0,05$.

\section{RESULTADOS}

Participaram do estudo 924 crianças e adolescentes entre seis e 13 anos de idade, sendo 474 do sexo masculino. A tabela 1 apresenta as características da amostra. Observou-se que nas crianças (6-9 anos) as médias de PerC, RCE e ÍndiceC foram maiores no sexo masculino. As adolescentes do sexo feminino eram mais altas, e o PerC, a RCE e o ÍndiceC foram maiores no sexo masculino.

Tabela1. Características gerais da amostra.

\begin{tabular}{|c|c|c|c|c|c|c|}
\hline & \multicolumn{2}{|c|}{$\begin{array}{c}\text { Crianças } \\
(6-9 \text { anos })\end{array}$} & \multicolumn{4}{|c|}{$\begin{array}{l}\text { Adolescentes } \\
\text { (10-13 anos) }\end{array}$} \\
\hline & $\begin{array}{l}\text { Masculino } \\
(n=273)\end{array}$ & $\begin{array}{c}\text { Feminino } \\
(n=250)\end{array}$ & p-valor & $\begin{array}{l}\text { Masculino } \\
(\mathrm{n}=201)\end{array}$ & $\begin{array}{c}\text { Feminino } \\
(n=200)\end{array}$ & p-valor \\
\hline & $\overline{\mathrm{x}}(\mathrm{dp})$ & $\overline{\mathrm{x}}(\mathrm{dp})$ & & $\overline{\mathrm{x}}(\mathrm{dp})$ & $\overline{\mathrm{x}}(\mathrm{dp})$ & \\
\hline Idade (anos) & $7,97(0,94)$ & $7,91(0,98)$ & 0,543 & $10,95(1,07)$ & $11,01(1,09)$ & 0,580 \\
\hline Massa corporal (kg) & $29,53(8,00)$ & $28,15(7,65)$ & 0,054 & $42,98(29,76)$ & $41,82(12,45)$ & 0,314 \\
\hline Estatura $(\mathrm{cm})$ & $129,20(7,43)$ & $127,90(8,03)$ & $0,055^{\dagger}$ & $144,58(10,40)$ & $146,51(9,61)$ & 0,030 \\
\hline IMC $\left(\mathrm{kg} / \mathrm{m}^{2}\right)$ & $17,50(3,60)$ & $17,04(3,31)$ & 0,112 & $20,11(12,13)$ & $19,24(4,40)$ & 0,900 \\
\hline $\operatorname{PerC}(\mathrm{cm})$ & $60,00(8,74)$ & $57,34(7,84)$ & $<0,001$ & $65,90(10,42)$ & $64,06(9,17)$ & 0,081 \\
\hline RCE & $0,46(0,06)$ & $0,45(0,06)$ & $<0,001$ & $0,46(0,06)$ & $0,44(0,06)$ & 0,001 \\
\hline ÍndiceC & $1,16(0,09)$ & $1,13(0,09)$ & $<0,001$ & $1,14(0,09)$ & $1,12(0,18)$ & $<0,001$ \\
\hline
\end{tabular}

IMC: índice de massa corporal; PerC: perímetro da cintura; RCE: razão cintura estatura; ÍndiceC: índice de conicidade; ̄̄: média; dp: desvio padrão; n: frequência absoluta; †teste t para amostras independentes. 
Na tabela 2 são apresentados os valores de área sobre a curva ROC, os pontos de corte, a sensibilidade e especificidade para os indicadores de obesidade central na detecção de excesso de peso. Todos os indicadores demonstraram capacidade discriminatória para exces- so de peso, com exceção do ÍndiceC em crianças do sexo masculino. Observou-se que o PerC e a RCE foram melhores preditores de excesso de peso em relação ao ÍndiceC em ambos os sexos, em crianças e adolescentes.

Tabela 2. Acurácia diagnóstica dos indicadores de obesidade central para predizer excesso de peso, estratificado em crianças e adolescentes para cada sexo.

\begin{tabular}{|c|c|c|c|c|c|}
\hline $\begin{array}{l}\text { Sexo } \\
\text { masculino }\end{array}$ & Curva ROC (IC95\%) & p-valor & Ponto de corte & $\begin{array}{l}\text { Sensibilidade } \\
\text { (IC95\%) }\end{array}$ & $\begin{array}{l}\text { Especificidade } \\
\text { (IC95\%) }\end{array}$ \\
\hline \multicolumn{6}{|l|}{ Crianças } \\
\hline PerC & $0,94(0,90-0,96)^{*}$ & $<0,001$ & 60,5 & $\begin{array}{c}89,7(80,8- \\
95,5)\end{array}$ & $86,7(81,1-91,1)$ \\
\hline RCE & $0,92(0,89-0,95)^{*}$ & $<0,001$ & 0,46 & $\begin{array}{c}89,7(80,8- \\
95,5)\end{array}$ & $81,5(75,4-86,7)$ \\
\hline ÍndiceC & $0,54(0,48-0,60)$ & 0,335 & 1,22 & $\begin{array}{c}26,9(17,5- \\
38,2)\end{array}$ & $90,8(85,8-94,4)$ \\
\hline \multicolumn{6}{|l|}{ Adolescentes } \\
\hline PerC & $0,95(0,91-0,98)^{*}$ & $<0,001$ & 66,5 & $\begin{array}{c}93,2(83,5- \\
98,1)\end{array}$ & $88,0(81,5-92,9)$ \\
\hline & $0,95(0,91-0,97)^{*}$ & $<0,001$ & 0,47 & $\begin{array}{c}88,1(77,1- \\
95,1)\end{array}$ & $91,5(85,7-95,6)$ \\
\hline & $0,74(0,67-0,80)^{*}$ & $<0,001$ & 1,15 & $\begin{array}{c}69,5(56,1- \\
80,8)\end{array}$ & $67,6(59,2-75,2)$ \\
\hline Sexo feminino & & & & & \\
\hline \multicolumn{6}{|l|}{ Crianças } \\
\hline PerC & $0,95(0,91-0,97)^{*}$ & $<0,001$ & 57,5 & $\begin{array}{c}91,4(91,0- \\
97,1)\end{array}$ & $84,4(78,5-89,2)$ \\
\hline RCE & $0,92(0,88-0,95)^{*}$ & $<0,001$ & 0,45 & $\begin{array}{c}91,4(81,1- \\
97,1)\end{array}$ & $81,8(75,6-87,0)$ \\
\hline ÍndiceC & $0,65(0,59-0,71)^{*}$ & $<0,001$ & 1,18 & $\begin{array}{c}37,9(25,5- \\
51,6)\end{array}$ & $89,1(83,8-93,1)$ \\
\hline \multicolumn{6}{|l|}{ Adolescentes } \\
\hline PerC & $0,95(0,91-0,98)^{*}$ & $<0,001$ & 63,0 & $\begin{array}{c}95,4(87,1- \\
99,0)\end{array}$ & $85,2(78,0-90,7)$ \\
\hline RCE & $0,95(0,91-0,98)^{*}$ & $<0,001$ & 0,44 & $\begin{array}{c}86,2(75,3- \\
93,5)\end{array}$ & $88,1(81,5-93,1)$ \\
\hline ÍndiceC & $0,63(0,56-0,70)^{*}$ & 0,002 & 1,09 & $\begin{array}{c}70,8(58,2- \\
81,4)\end{array}$ & $51,1(42,4-59,8)$ \\
\hline
\end{tabular}

PerC: perímetro da cintura; RCE: razão cintura estatura; ÍndiceC: índice de conicidade; IC95\%: intervalo de confiança de 95\%*área sobre a curva ROC que apresenta poder discriminatório para excesso de peso.

Foram observadas diferenças significativas entre as áreas sobre a curva ROC, indicando que a curva ROC do ÍndiceC tem o menor percentual abaixo da curva quando comparado ao PerC e a RCE ( $<<0,001)$ em todas as análises (Figuras 1 , $2,3$ e 4$)$. 


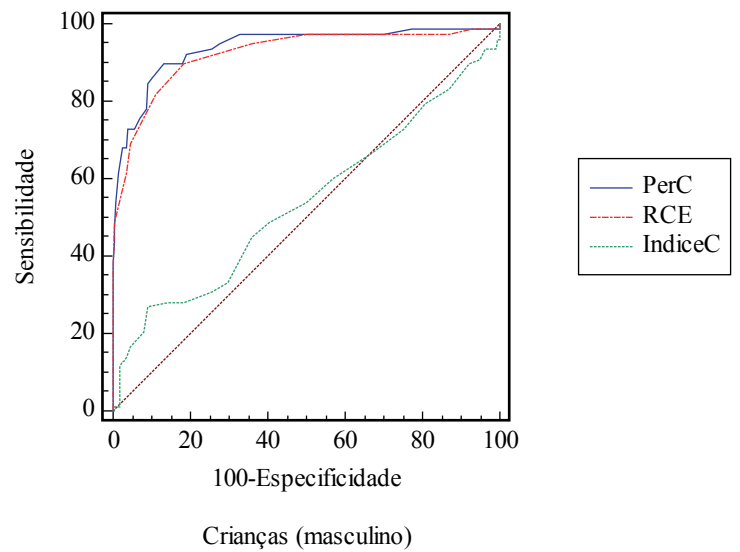

Figura 1. Áreas sobre a curva ROC de indicardores de obesidade central para predizer excesso de peso em crianças sexo masculino.

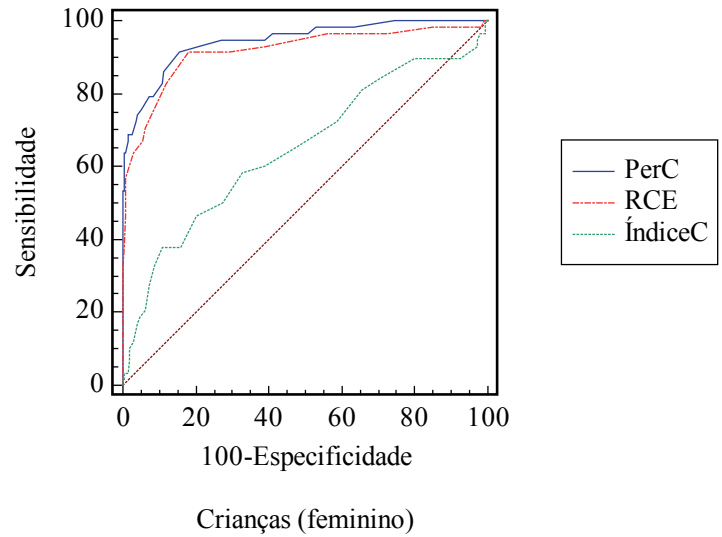

Figura 3. Áreas sobre a curva ROC de indicardores de obesidade central para predizer excesso de peso em crianças sexo feminino.

\section{DISCUSSÃo}

Este estudo verificou a capacidade de indicadores de obesidade central em predizer o excesso de peso de crianças e adolescentes de ambos os sexos. Com exceção do ÍndiceC em crianças do sexo masculino, o qual apresentou o valor inferior do IC $95 \%$ da área sobre a curva ROC < 0,5, todos os indicadores de obesidade central demonstraram capacidade de discriminar excesso de peso em crianças e adolescentes.

Poucos estudos investigaram a capacidade de indicadores antropométricos como preditores de excesso de peso (ANWAR et al., 2019; BATISTA et al., 2019; KNABBEN et al., 2015). Anwar et al. (2019) verificaram que o perímetro da

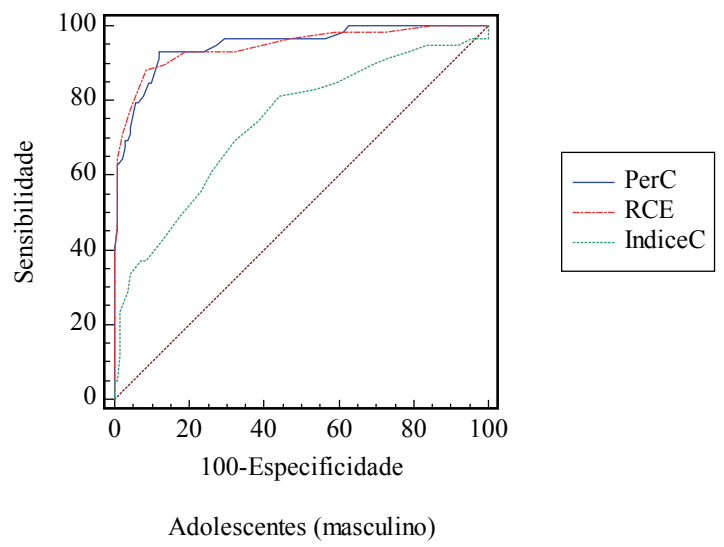

Figura 2. Áreas sobre a curva ROC de indicardores de obesidade central para predizer excesso de peso em adolescentes do sexo masculino.

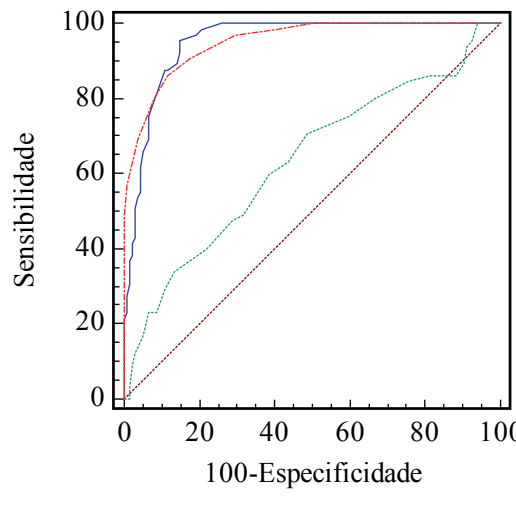

PerC
- RCE
ÍndiceC

Figura 4. Áreas sobre a curva ROC de indicardores de obesidade central para predizer excesso de peso em adolescentes do sexo feminino.

cintura e a razão cintura estatura apresentaram boa área sobre a curva ROC para predizer excesso de peso em adultos de Rustaque, em Omã. Os autores verificaram que os melhores pontos de corte para perímetro de cintura foram de $89,5 \mathrm{~cm}$ e $87,6 \mathrm{~cm}$ para homens e mulheres, respectivamente, e para razão cintura estatura foram de 0,53 e 0,57 respectivamente para homens e mulheres.

Um estudo conduzido com 254 adolescentes de 12 a 17 anos de Rio do Sul, Santa Catarina, verificou boa capacidade do perímetro da cintura em discriminar excesso de peso determinado pelo percentil 85 do IMC (KNABBEN et al., 2015). Os pontos de corte encontrados 
pelos autores foram superiores aos do presente estudo, entretanto, deve-se levar em consideração o tamanho da amostra inferior e a faixa etária de adolescentes mais ampla em relação ao presente estudo.

Para crianças do sexo masculino e feminino os resultados do presente estudo indicaram os pontos de corte de perímetro da cintura de 60,5 e 57,5, respectivamente, como preditores de excesso de peso e 66,5 e 63,0 para adolescentes do sexo masculino e feminino, respectivamente. Weiss et al. (2016) investigaram adolescentes de 11 a 14 anos, estudantes de escolas públicas e privadas de Florianópolis, Santa Catarina e encontraram pontos de corte de perímetro de cintura de 68,7 para o sexo masculino e 65,9 para o sexo feminino, os quais demonstraram boa capacidade de predizer gordura corporal elevada, determinada pelo somatório de dobras cutâneas da região de tríceps e panturrilha.

Outro estudo realizado com crianças de Florianópolis (7 a 10 anos) também identificou indicadores antropométricos de obesidade como bons preditores de gordura corporal elevada (RIBEIRO; LEAL; ASSIS, 2014). Os pontos de corte propostos pelos autores foram semelhantes aos encontrados no presente estudo, apesar de terem avaliado a gordura corporal, diferentemente do presente estudo que avaliou o excesso de peso. No sexo masculino, a crianças de 7 a 9 anos de Florianópolis (RIBEIRO; LEAL; ASSIS, 2014) apresentaram pontos de corte para perímetro da cintura que variaram de 59,1 a 63,2 , já os estudantes de 10 anos, apresentaram ponto de corte de 66,4, mais próximo ao ponto de corte identificado para adolescentes do sexo masculino no presente estudo $(66,5)$. No sexo feminino, o ponto de corte das crianças de Florianópolis variou de 56,8 a 61,5, também próximos aos encontrados no presente estudo $(57,5)$.

Os pontos de corte de RCE para discriminar excesso de peso em crianças e adolescentes do sexo masculino foram de 0,46 e 0,47, respectivamente, e para crianças e adolescentes do sexo feminino foram de 0,45 e 0,44, respectivamente. Num estudo realizado com crianças e adolescentes de 9 a 12 anos do município de Tubarão, Santa Catarina, os autores identificaram pontos de corte de RCE de 0,43 para discriminar sobrepeso e 0,45 para discriminar obesidade em ambos os sexos (BATISTA et al., 2019). Da mesma forma como foi encontrado no presente estudo, esses pontos de corte são inferiores aos recomendados internacionalmente por Ashwell e
Hsieh (2005), os quais propõem o ponto de corte de 0,5 para RCE elevada independentemente de idade, sexo e etnia. 0 presente estudo corrobora outros estudos que também verificaram pontos de corte para RCE inferiores a 0,5 para crianças e adolescentes, utilizando métodos de avaliação de composição corporal mais precisos como critério de referência para análise de curva ROC (PELEGRINI et al., 2015; WEISS et al., 2016).

O ÍndiceC demonstrou a pior capacidade de diagnóstico de excesso de peso em relação ao PerC e a RCE, com pontos de corte de 1,15 para adolescentes do sexo masculino e no sexo feminino 1,18 para crianças e 1,09 para adolescentes. Em adolescentes de 11 a 14 anos do sexo masculino de Florianópolis, o ÍndiceC apresentou boa capacidade de predizer gordura corporal elevada com ponto de corte de 1,13 (WEISS et al., 2016). Em adolescentes de 15 a 17 anos o ponto de corte de ÍndiceC para predizer gordura corporal elevada foi de 1,12 para o sexo masculino e 1,06 para o sexo feminino (PELEGRINI et al., 2015). Embora o ÍndiceC tenha demonstrado ser um bom preditor de gordura corporal elevada e excesso de peso, seu desempenho é inferior a outros indicadores de obesidade central, como o PerC e RCE (WEISS et al., 2016; PELEGRINI et al., 2015).

Recomenda-se que os professores de educação física realizem a avaliação da composição corporal dos escolares no início e término do ano letivo. A partir dessas avaliações pode-se acompanhar o crescimento das crianças e adolescentes e realizar a identificação precoce de possíveis fatores de risco para doenças (BECK et al., 2007). Para isso os indicadores antropométricos são considerados uma opção simples e confiável para o monitoramento do estado nutricional de crianças e adolescentes (PELEGRINI et al., 2015).

Deve-se levar em consideração que o IMC apresenta várias limitações como indicador de obesidade, por não diferenciar a massa gorda da massa magra (BUSS, 2014). Entretanto, além de ser recomendado pela OMS (WHO, 2000) como indicador para determinar sobrepeso e obesidade, o IMC é considerado um indicador razoavelmente bom na identificação de gordura corporal (JENSEN; CAMARGO; BERGAMASCHI et al., 2016) e apresenta boa relação com outros desfechos negativos em saúde, como risco de doenças cardiometabólicas (BLÜHER, et al., 2013). Sabe-se que o acúmulo de gordura depositado na região central do corpo representa 
maior risco de saúde em relação à gordura periférica (FOX et al., 2007), neste sentido, os indicadores de obesidade central podem ser utilizados para identificar crianças e adolescentes com excesso de peso a partir dos pontos de corte propostos nesse estudo. Estes indicadores de obesidade central apresentam como vantagem o baixo custo, visto que necessitam apenas da medida de perímetro da cintura e de estatura, que podem ser mensurados por uma fita métrica, a qual pode ser adquirida por um preço mais acessível em relação a uma balança digital.

\section{CONCLUSÕES}

Os resultados do presente estudo revelaram que indicadores antropométricos de obesidade central possuem boa capacidade para predizer excesso de peso em crianças e adolescentes de ambos os sexos. Sugere-se que o PerC e a RCE sejam utilizados na avaliação do estado nutricional, por professores de educação física e profissionais da saúde, para identificar crianças e adolescentes com risco de saúde. Estes indicadores são facilmente mensurados e de baixo custo, uma vez que apenas uma fita métrica pode fornecer as medidas necessárias para determinar os indicadores. 


\section{REFERÊNCIAS}

ABARCA-GÓMEZ, L. et al. Worldwide trends in body-mass index, underweight, overweight, and obesity from 1975 to 2016 : a pooled analysis of 2416 population-based measurement studies in 128.9 million children, adolescents, and adults. The Lancet, v. 390, n. 10113, p. 2627-2642, 2017.

ALVES JUNIOR, C. A. S. et al. Anthropometric indicators as body fat discriminators in children and adolescents: a systematic review and meta-analysis. Advances in nutrition, v. 8, n. 5, p. 718-727, 2017.

ANWAR, S. et al. Which is a better marker for overweight: waist height ratio or waist circumference? International Journal of Research in Medical Sciences, v. 7, n. 2, p. 462, 2019.

BATISTA, E. et al. Acurácia da relação cintura-altura comparada ao índice de massa corpórea no diagnóstico de sobrepeso e obesidade infantil. ACM arq. catarin. med, 2019.

BECK, C. C. et al. Ficha antropométrica na escola: o que medir e para que medir. Revista Brasileira de Cineantropometria e Desempenho Humano, v. 9, n. 1, p. 107-14, 2007.

BLÜHER, S. et al. Body mass index, waist circumference, and waist-to-height ratio as predictors of cardiometabolic risk in childhood obesity depending on pubertal development. The Journal of Clinical Endocrinology \& Metabolism, v. 98, n. 8, p. 3384-3393, 2013.

BUSS, J. Limitations of body mass index to assess body fat. Workplace health \& safety, v. 62, n. 6, p. 264-264, 2014.

CARVALHO, M. S. N. et al. Accuracy of Anthropometric Indices in Predicting Excess Abdominal Adiposity among Ten to Eighteen-Year-Old Adolescents. Journal of Exercise Physiology Online, v. 21, n. 4, 2018.

CERDA, J.; CIFUENTES, L. Uso de curvas ROC en investigación clínica: Aspectos teórico-prácticos. Revista chilena e infectologia, Santiago, v. 29, n. 2, p. 138-141, abr. 2012 .

COLE, T. J. et al. Establishing a standard definition for child overweight and obesity worldwide: international survey. Bmj v. 320 , n. 7244 , p. $1240,2000$.

DUMITH, S. C. et al. Diagnostic properties and cutoff points for overweight prediction through anthropometric indicators in adolescents from Caracol, Piauí, Brazil, 2011. Epidemiologia e Serviços de Saúde, v. 27, p. e201715013, 2018.

FERNANDES FILHO, J. A prática da avaliação física: testes, medidas e avaliação física em escolares, atletas e academias de ginástica. 2. ed. Rio de Janeiro: Shape, 2003.

FOX, C. S. et al. Abdominal visceral and subcutaneous adipose tissue compartments. Circulation, v. 116, n. 1, p. 39-48, 2007

JENSEN, N. S. O.; CAMARGO, T. F. B.; BERGAMASCHI, D. P. Comparison of methods to measure body fat in 7-to-10-year-old children: a systematic review. Public Health, v. 133, p. 3-13, 2016.

KNABBEN, E. G. et al. Valor de referência da circunferência da cintura a partir do percentil 85 do "imc" de adolescentes. Biomotriz, v. 8, n. 2, 2015.

KUCIENE, R; DULSKIENE, V. Associations between body mass index, waist circumference, waist-to-height ratio, and high blood pressure among adolescents: a cross-sectional study. Scientific reports, v. 9, n. 1, p. 1-11, 2019.

MINISTÉRIO DA SAÚDE; CONSELHO NACIONAL DE SAÚDE. Resolução no 466, de 12 de dezembro de 2012. Diretrizes e normas regulamentadoras de pesquisas envolvendo seres humanos. Diário Oficial da União [da] República Federativa do Brasil, v. 150, n. 112, 2013.

RIBEIRO, E. A. G.; LEAL, D. B.; ASSIS, M. A. A. Acurácia diagnóstica de índices antropométricos na predição do excesso de gordura corporal em crianças de sete a dez anos. Rev. Bras. Epidemiol., São Paulo, v. 17, n. 1, p. 243-254, Mar. 2014.

SIMMONDS, M. et al. Predicting adult obesity from childhood obesity: a systematic review and meta-analysis. Obesity Reviews, v. 17, n. 2, p. 95-107, 2016.

WEISS, K. M. et al . Diagnostic accuracy of anthropometric indicators to predict excess body fat in adolescents aged 11-14 years. Revista Brasileira de Cineantropometria e Desempenho Humano, Florianópolis, v. 18, n. 5, p. 548-556, 2016.

WORLD HEALTH ORGANIZATION. Obesity: preventing and managing the global epidemic. World Health Organization, 2000.

WORLD HEALTH ORGANIZATION et al. Nutrition in adolescence: issues and challenges for the health sector: issues in adolescent health and development. 2005. 